\title{
Exact low-temperature behavior of kagomé antiferromagnet at high fields
}

\author{
M. E. Zhitomirsky \\ Commissariat à l'Energie Atomique, DSM/DRFMC/SPSMS, 38054 Grenoble, France \\ Hirokazu Tsunetsugu \\ Yukawa Institute for Theoretical Physics, Kyoto University, Kyoto 606-8502, Japan
}

(Dated: May 25, 2004)

\begin{abstract}
Low-energy degrees of freedom of a spin-1/2 kagomé antiferromagnet in the vicinity of the saturation field are mapped to a hard-hexagon model on a triangular lattice. The latter model is exactly solvable. The presented mapping allows to obtain quantitative description of the magnetothermodynamics of a quantum kagomé antiferromagnet up to exponentially small corrections as well as predict the critical behavior for the transition into a magnon crystal state. Analogous mapping is presented for the sawtooth chain, which is mapped onto a model of classical hard dimers on a chain.

PACS numbers: $75.50 . \mathrm{Ee}, 75.45 .+\mathrm{j}, 75.10 . \mathrm{Jm}, 75.30 . \mathrm{Sg}$
\end{abstract}

In the past few years there have been increased attention to the behavior of frustrated magnetic materials at high fields. The interest is stimulated by a search for new fundamental effects such as the magnetization plateaus,$\frac{1.2 .3 .4 .5 .6 .7 .8 .9}{1}$ the magnetization jumps new complicated magnetic phase diagrams,,$\frac{8,14}{,}$ and new types of the critical behavior $\frac{15}{15}$ as well as by a possible technological application of frustrated magnets in the adiabatic demagnetization refrigerators due to their unique magnetocaloric properties $\stackrel{16,17}{ }$ A zerotemperature behavior of geometrically frustrated antiferromagnets in the vicinity of the saturation field $H_{c}$ has been recently discussed by Schulenburg and coworkers 10.11 .12 .13 Above $H_{c}$ in the fully polarized phase, these models have a flat branch of magnons with energy $\left(H-H_{c}\right)$. Such dispersionless excitations correspond in real space to localized spin flips, which do not interact with each other unless touched. Upon decreasing magnetic field through $H_{c}$, the energy of a single excitation becomes negative and magnons condense into a closepacked crystal structure. The present work is devoted to theoretical investigation of the low-temperature thermodynamics of magnon crystals. Specifically, we consider spin-1/2 Heisenberg models on a kagomé lattice and a sawtooth ( or $\Delta$ ) chain, see Fig. 11 While the former lattice is one of the best known examples of geometric frustration, which is realized, e.g., in $\mathrm{SrCr}_{9 p} \mathrm{Ga}_{12-9 p} \mathrm{O}_{19}{ }_{18}^{18}$ the latter spin model is applicable to magnetic delafossite $\mathrm{YCuO}_{2.5} \stackrel{19}{=}$ We show that the thermodynamic potential in the vicinity of $H_{c}$ for the two quantum models can be calculated exactly up to corrections, which are exponentially small at low temperatures.

We consider nearest-neighbor spin-1/2 Heisenberg models in an external field

$$
\hat{\mathcal{H}}=\sum_{\langle i j\rangle} J_{i j} \mathbf{S}_{i} \cdot \mathbf{S}_{j}-\mathbf{H} \cdot \sum_{i} \mathbf{S}_{i}
$$

For a kagomé lattice all nearest-neighbor bonds have the same strength $J_{i j} \equiv 1$. The spectrum of single-magnon excitations in the fully polarized phase is

$$
\omega_{1 \mathbf{k}}=H-3, \omega_{2,3 \mathbf{k}}=H-\frac{3}{2} \pm \frac{1}{2} \sqrt{3\left(1+2 \gamma_{\mathbf{k}}\right)},
$$

where $\gamma_{\mathbf{k}}=\frac{1}{6} \sum_{\delta} e^{i \mathbf{k} \cdot \delta}$ is a sum over nearest-neighbor sites on a triangular Bravais lattice. At the saturation field $H_{c}=3$ the energy of excitations from the lowest dispersionless branch vanishes. The sawtooth chain exhibits a similar behavior for the special ratio of the two coupling constants $J_{2}=\frac{1}{2} J_{1}$. In the following we always assume the above choice of the parameters with $J_{1} \equiv 1$. Above $H_{c}=2$ the sawtooth chain antiferromagnet has two branches of single-magnon excitations:

$$
\omega_{1 k}=H-2, \quad \omega_{2 k}=H-\frac{1}{2}(1-\cos k) .
$$

Excitations in the dispersionless branches correspond to localized states. Simplest examples of such states are spin flips trapped on hexagon voids of a kagomé lattice or in the valleys of the sawtooth chain, see Fig. 1 Their wave functions are given by

$$
\begin{aligned}
& \left|\varphi_{i}\right\rangle=\frac{1}{\sqrt{6}} \sum_{n=1}^{6}(-1)^{n-1} S_{n i}^{-}|\mathrm{FM}\rangle, \\
& \left|\varphi_{i}\right\rangle=\frac{1}{\sqrt{6}}\left(S_{1 i}^{-}-2 S_{2 i}^{-}+S_{3 i}^{-}\right)|\mathrm{FM}\rangle,
\end{aligned}
$$

for the kagomé and the sawtooth models, respectively, $|F M\rangle$ being the ferromagnetic state. Other more extended localized states can be represented as linear combinations of the above elementary states. Moreover, for a kagomé lattice with $N$ sites, there is the same number of hexagons $\frac{1}{3} N$ as the number of states in the flat branch. Therefore, the localized states on the hexagons states can be used as a real-space basis for the lowest branch in Eq. (2). Similar correspondence exists between the $\frac{1}{2} N$ states in the dispersionless branch Eq. (3) and the localized states in the valleys of the sawtooth chain.

Localized one-magnon states allow to construct a class of exact eigenstates in every $n$-magnon subsector with $n \leq N_{\max }$ by putting localized spin flips on isolated 


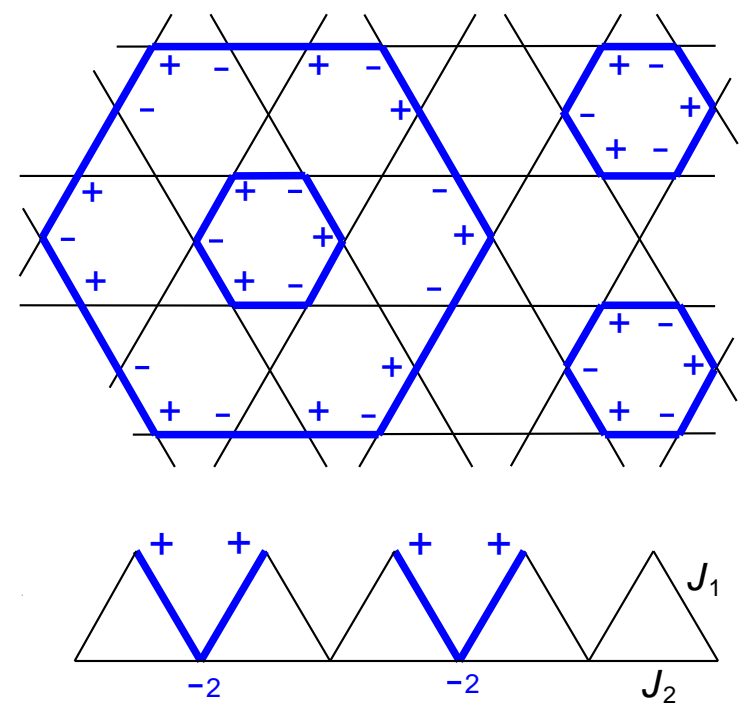

FIG. 1: (color online) Kagomé lattice (top) and the sawtooth chain (bottom) with localized magnons. Amplitudes and phases of spin-down states are indicated near each site.

hexagons or valleys. The highest possible density of independent localized magnons on the kagomé lattice is onethird of the total number of hexagons or $N_{\max }=\frac{1}{9} N$. Such a magnon crystal state is three-fold degenerate and breaks the translational symmetry. A magnon crystal for the sawtooth chain has $N_{\max }=\frac{1}{4} N$ and is two-fold degenerate. Since isolated localized magnons do not interact with each other, it is natural that they correspond to the lowest energy states in every $n$-magnon subsector $\left(n \leq N_{\max }\right)^{10,11,12.13}$ Our idea is that only isolated localized magnons contribute to the low temperature thermodynamics. In order to prove this we have to show that (i) there are no other low energy states and that (ii) isolated localized magnon states are separated by a finite gap from the higher-energy states in the same $S^{z}$ subsector. In the following we first prove the above two points and then show that localized magnons are mapped to statistical mechanics models of hard classical objects, hexagons or dimers, which allow exact solution for the thermodynamic properties.

Numerical evidences and analytical arguments that isolated localized magnon states are the lowest energy states in every $n$-magnon sector have been presented before ${ }^{10.11 .12 .13}$ Below we give a shortened proof for the spin-1/2 kagomé antiferromagnet, which is also valid for the checkerboard and the pyrochlore lattice antiferromagnets. The Heisenberg Hamiltonian (11) is split in a standard way into the Ising part $\hat{\mathcal{H}}^{z z}$, which includes all $S_{i}^{z} S_{j}^{z}$ terms plus the Zeeman energy, and the transverse part $\hat{\mathcal{H}}^{\perp}$. The energy of the ferromagnetic state is always subtracted from $\hat{\mathcal{H}}^{z z}$. A quantum state of $n$ isolated localized magnons is not only an eigenstate of $\hat{\mathcal{H}}$ with $E_{n}=n(H-3)$, but is also an eigenstate of $\hat{\mathcal{H}}^{z z}$ and $\hat{\mathcal{H}}^{\perp}$ separately with $E_{n}^{z z}=n(H-2)$ and $E_{n}^{\perp}=-n$. The idea is to show that for an arbitrary state $\left\langle\hat{\mathcal{H}}^{z z}\right\rangle \geq E_{n}^{z z}$ and $\left\langle\hat{\mathcal{H}}^{\perp}\right\rangle \geq E_{n}^{\perp}$. For $\hat{\mathcal{H}}^{z z}$ the formulated relation holds trivially: once all spin flips sit on different bonds the Ising part of the Hamiltonian has the minimal energy $E_{n}^{z z}$. In order to show that the same is true for the transverse part we map the subspace of $n$ spin flips on the Hilbert space of $n$ hard-core bosons $\mathcal{B}_{n}$. The transverse part of the Heisenberg Hamiltonian is, then, the kinetic energy of bosons $\hat{\mathcal{H}}^{\perp} \equiv \hat{\mathcal{K}}$. In addition, we define the Hilbert space of $n$ bosons without the hard-core constraint: $\mathcal{B}_{0 n} \supset \mathcal{B}_{n}$. The minimum of the kinetic energy is easily found in $\mathcal{B}_{0 n}$ : all $n$ particles should occupy one of the low-energy singleparticle states given by Eq. (2). If the allowed quantum states are restricted again to $\mathcal{B}_{n}$, from the variational principle the expectation value of $\hat{\mathcal{K}}$ can only increase: $\left\langle\hat{\mathcal{H}}^{\perp}\right\rangle \geq \min _{\mathcal{B}_{0 n}}\langle\hat{\mathcal{K}}\rangle=E_{n}^{\perp}$. The presented arguments not only show that isolated localized magnons have the lowest energy, but they also allow to check whether these states are the only lowest energy states (see below).

For the sawtooth chain the two branches of excitations in Eq. (3) are separated by a gap. The low-energy scattering states are, therefore, formed by magnons from the flat branch. In the two-magnon sector there are $\frac{1}{2}(N / 2)(N / 2+1)$ states constructed from $N / 2$ onemagnon states of the lowest branch. The number of isolated localized magnons in this sector is $\frac{1}{2}(N / 2)(N / 2-3)$. The difference gives the number of scattering states, which is equal to $N$. In real space representation the above scattering states correspond to localized magnons occupying the same valley or adjacent valleys. This representation suggests the following basis in the subspace of scattering states: $\left|\psi_{i}\right\rangle=\left|\varphi_{i} \varphi_{i+1}\right\rangle$ and $\left|\widetilde{\psi}_{i}\right\rangle=\left|\varphi_{i}^{2}\right\rangle$, where the exclusion principle of spin-flips and proper normalization are implied. These states have nonzero overlaps:

$$
\left\langle\psi_{i} \mid \psi_{i \pm 1}\right\rangle=1 / 35, \quad\left\langle\psi_{i} \mid \widetilde{\psi}_{i}\right\rangle=\left\langle\psi_{i} \mid \widetilde{\psi}_{i+1}\right\rangle=\sqrt{5 / 49} .
$$

We calculate the matrix elements of the Hamiltonian between these states and use the variational principle to determine the lowest possible energy. A simple variational state $|k\rangle=\sum_{i} e^{-i k r_{i}}\left|\psi_{i}\right\rangle$ has the energy $E(k)=$ $\langle k|\hat{\mathcal{H}}| k\rangle /\langle k \mid k\rangle$. The minimal value of $E(k)$ is reached at $k=0$ with the gap $\Delta=\frac{20}{37} \approx 0.54$, which separates the two-magnon scattering states from the low-energy boundary $2(H-2)$. An improved variational ansatz $|k\rangle=\sum_{i} e^{-i k r_{i}}\left(\left|\psi_{i}\right\rangle+c\left|\widetilde{\psi}_{i}\right\rangle\right)$ yields $\Delta \approx 0.44$, which compares well with the exact diagonalization result $0.42,20$ The gap is determined mostly by a nearest-neighbor repulsion of localized magnons in adjacent valleys. The dispersion of bound two-magnon complexes $E(k)$ is weak and does not exceed $10 \%$. We conjecture that the repulsion leads to a similar behavior in all $n$-magnon sectors: the lowest energy states with $E_{n}=n(H-2)$ are separated from the scattering states by a finite gap $\Delta_{n}=O(1)$. At temperatures $T \ll \Delta_{n}$ one can neglect the higher energy states and consider only contribution of isolated localized magnons. The latter problem is equivalent to a one-dimensional lattice gas of particles with 
energies $(H-2)$ and on-site and nearest-neighbor exclusion principle (classical hard-dimer model).

The above consideration is straightforwardly extended to the kagomé antiferromagnet. The two-magnon sector consists of $\frac{1}{2}(N / 3)(N / 3+1)$ states constructed from one-magnon states of the flat branch $\omega_{1 \mathbf{k}}$. They include $\frac{1}{2}(N / 3)(N / 3-7)$ states constructed by placing localized spin flips on isolated hexagons (called below hardhexagon states) and the additional $\frac{4}{3} N$ defect states. The latter states correspond to $N$ states of localized magnons, which occupy adjacent hexagons, and to $\frac{1}{3} N$ states with two spin flips on the same hexagon. We define the realspace basis for the defect states as $\left|\psi_{\alpha i}\right\rangle=\left|\varphi_{i} \varphi_{i+\delta_{\alpha}}\right\rangle$ and $\left|\widetilde{\psi}_{i}\right\rangle=\left|\varphi_{i}^{2}\right\rangle$, where $\delta_{1}=(1,0)$ and $\delta_{2,3}=\left(\frac{1}{2}, \pm \frac{\sqrt{3}}{2}\right)$ are three nearest-neighbor sites on a triangular lattice of hexagons. Using a simple variational ansatz $|\mathbf{k}\rangle=$ $\sum_{i \alpha} e^{-i \mathbf{k r}_{i}} c_{\alpha}\left|\psi_{\alpha i}\right\rangle$ we find the gap $\Delta \approx 0.24$ between the isolated localized states and the scattering defect states.

The low-energy sector of the kagomé antiferromagnet has also two additional features. First, the hard-hexagon states are not the only independent localized magnon states. An extra two-magnon state is illustrated in the left part of Fig. 1 and consists of two spin flips cycling around a small and a large hexagons. Such a state is a special combination of the defect states $\left|\psi_{\alpha i}\right\rangle$ and $\left|\widetilde{\psi}_{i}\right\rangle$. Combinatorial arguments show that the extra states give only $1 / N$ corrections to the hard-hexagon result in $n$ magnon sectors with small $n$ and disappear completely in sectors with $3 n / N \gtrsim 0.1$

Second difference with the sawtooth chain is that the gap for one of the dispersive branches in Eq. (2) also vanishes at $H=H_{c}$. The corresponding propagating magnon has the same energy $\omega_{3 \mathbf{k}=0}=H-3$ as localized magnons from the flat branch. The ground states in the two-magnon sector contain apart from the isolated localized magnons also superposition states of one localized magnon and one propagating magnon. Such states form a continuum above the low-energy threshold $E_{2}=2(H-3)$. The same is true for all $n$-magnon sectors with $n \ll N$. Once $n$ becomes a finite fraction of $N$, i.e. for partially magnetized states, the above picture changes. The low-energy propagating magnons experience multiple scattering from an infinite number of localized magnons. Such an interaction produces a finite shift of their energy. The exact value of the energy shift for the $\omega_{3 \mathbf{k}}$ depends on a precise pattern of localized magnons. It can be explicitly estimated for a 'uniform' state of $n$-localized magnons, corresponding to a low-temperature ensemble of states with different translational patterns. We bosonize the spin Hamiltonian using, e.g., the Holstein-Primakoff transformations. The quadratic terms give the excitation spectrum, which coincides with Eq. (2). To treat the effect of interactions we define two Hartree-Fock averages $m_{1}=\left\langle b_{i}^{\dagger} b_{i}\right\rangle$ and $m_{2}=\left\langle b_{i}^{\dagger} b_{j}\right\rangle$. After decoupling the fourboson terms the effect of interaction is reduced to the renormalization $S \rightarrow\left(S-m_{1}+m_{2}\right)$ in the quadratic terms, where $S=1 / 2$ is a local spin. At the saturation field $H_{c}=6 S=3$ the gap for the dispersive mode is $\Delta_{d}=6\left(m_{1}-m_{2}\right)$. For a single localized magnon one finds $m_{1}=-m_{2}=\frac{1}{6}$. Therefore, the dispersive mode opens a finite gap $\Delta_{d}=3 n / N$. At low temperatures we neglect these higher-energy states and reduce the isolated localized magnons on small hexagons to a gas of particles on a triangular lattice with on-site and nearest-neighbor exclusion, which is also called a hard-hexagon model. A remarkable feature of this model is that it has an exact solution, which was obtained by Baxter ${ }^{21}$ Below, we discuss the low temperature properties of the kagomé antiferromagnet and the sawtooth chain in the framework of the two exactly solvable models.

The partition function of the classical hard dimers is

$$
Z=\sum_{\{\sigma\}} \exp \left[-\frac{\varepsilon}{T} \sum_{i} \sigma_{i}\right] \prod_{\langle i j\rangle}\left(1-\sigma_{i} \sigma_{j}\right),
$$

where $\varepsilon=H-H_{c}$ is the chemical potential and $\sigma_{i}=0,1$ are the occupation numbers for dimers the index $i$ runs over the $N / 2$ sites of the basal chain. The nearestneighbor exclusion principle is imposed by the last term. The partition function (6) can be rewritten in terms of the transfer matrices $\mathcal{T}\left(\sigma_{i}, \sigma_{i+1}\right)$ as $Z=\operatorname{Tr} \mathcal{T}^{N / 2}$. In the thermodynamic limit $N \rightarrow \infty$ the free energy is determined by the largest eigenvalue of the transfer matrix:

$$
F / N=-\frac{1}{2} T \ln \left(\frac{1}{2}+\sqrt{\frac{1}{4}+e^{-\varepsilon / T}}\right) .
$$

From this expression one can calculate various thermodynamic properties of the sawtooth chain. At $H=H_{c}$ the entropy and the magnetization have universal values independent of temperature:

$$
S / N=\frac{1}{2} \ln \left(\frac{1+\sqrt{5}}{2}\right), \quad M / N=\frac{1+\sqrt{5}}{4 \sqrt{5}} .
$$

The entropy as a function of field reaches a sharp maximum at $H=H_{c}$, which amounts to $34.7 \%$ of the total entropy $N \ln 2$ of the sawtooth chain. At low temperatures all the magnetization curves $M(H)$ cross at $H=H_{c}$. Comparison between analytical and numerical exact diagonalization results shows a nice agreement below $T^{*} \approx 0.1 \stackrel{20}{2}$ The correlation length of the hard dimers is obtained from the ratio of the two eigenvalues of $\mathcal{T}$, and the result is

$$
\xi^{-1}=\ln \frac{\sqrt{1+4 e^{-\varepsilon / T}}+1}{\sqrt{1+4 e^{-\varepsilon / T}}-1},
$$

with the wavenumber $\pi$.

The partition function of the hard-hexagon model is given by the same expression (6), where the site index runs over triangular lattice formed by the centers of hexagon voids of the original kagomé lattice. This model was solved by Baxter, who used a corner transfer matrix method to obtain $Z$ as a function of the fugacity 


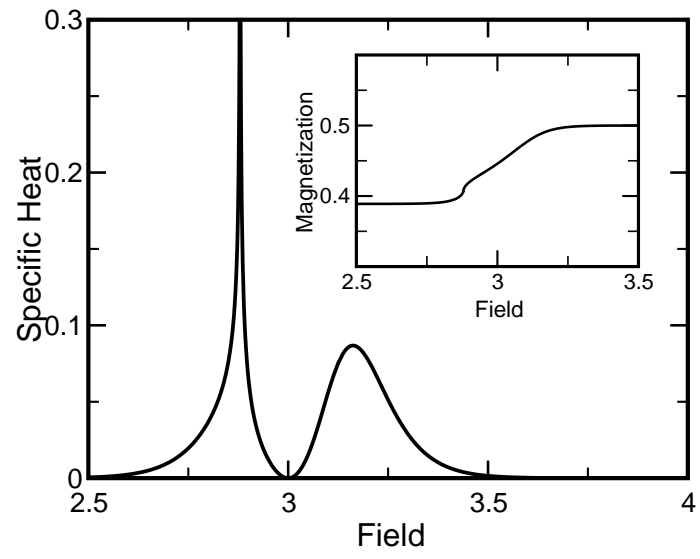

FIG. 2: Field dependence of the specific heat per one site (in units of $k_{B}$ ) of a spin-1/2 kagomé antiferromagnet at $T=0.05$ obtained in the hard-hexagon model. The inset shows the magnetization at the same $T$.

$z=e^{-\varepsilon / T, 21} \mathrm{~A}$ number of exact results following from the Baxter's solution can be transferred to the spin- $1 / 2$ kagomé antiferromagnet using a trivial rescaling. The entropy as a function of field at constant $T$ reaches a maximum at $H=H_{c}$. At the saturation field both the entropy and the magnetization have temperature independent universal values:

$$
S / N=0.11108, \quad M / N=0.44596 .
$$

More important results concern a phase transition, which corresponds to a weak crystallization of hard hexagons or localized magnons in the two models:

$$
\begin{aligned}
& H_{c}(T)=H_{c}-T \ln z_{c}, \quad z_{c}=(11+5 \sqrt{5}) / 2 \\
& M_{c} / N=\frac{1}{2}-\frac{1}{3} \rho_{c}, \quad \rho_{c}=(5-\sqrt{5}) / 10
\end{aligned}
$$

The second order transition at $H_{c}(T)$ belongs to the universality class of the three-state Potts model and has the exact critical exponents $\alpha=\frac{1}{3}, \beta=\frac{1}{9}$, and $\nu=\frac{5}{6}$. The transition field has a linear temperature dependence, while the magnetization $M_{c}$ stays along the critical line. Variations of the specific heat and the magnetization of the spin-1/2 kagomé antiferromagnet derived from the hard-hexagon model ${ }^{21}$ are presented in Fig. 2] Baxter used the order parameter defined as a difference of hexagon densities $\rho_{i}$ on adjacent sites: $R=\rho_{i}-\rho_{i+\delta}$. Up to a renormalization prefactor $R$ is related to the Fourier harmonics $\rho_{\mathbf{q}}$ at $\mathbf{q}=\left(4 \pi / 3 a^{*}, 0\right)$, where $a^{*}$ is a period of the triangular lattice. The magnon crystal has two types of spins, on hexagons with localized magnons and between them, which have different average magnetizations: $\left\langle S^{z}\right\rangle=1 / 3$ and $1 / 2$ at $T=0$, respectively. Hence, the crystalline order of magnons is reflected in appearance of the corresponding Fourier harmonics in the structure factor $S^{z z}(\mathbf{q})$ and can be in principle observed by elastic neutron scattering.

In conclusion, the above analysis of the magnetothermodynamics of the quantum kagomé antiferromagnet and of the sawtooth chain model has shown that strongly correlated spin systems have emergent "paramagnetic" degrees of freedom, which experience an effective magnetic field $h=H-H_{c}$. Such effective "paramagnetic moments" are responsible for an enhanced magnetocaloric effect of quantum geometrically frustrated antiferromagnets in the vicinity of the saturation field very similar to their classical counterparts 16 During preparation of the manuscript, we have learned about a recent preprint, $\stackrel{22}{,}$ where the authors have obtained a zerotemperature entropy of the two models in agreement with our Eqs. (8) and (10), though they have not discussed the low-temperature thermodynamics.

We thank G. Jackeli and A. Honecker for useful discussions. Stay of M.E.Z. at the YITP of Kyoto University was supported by a Grant-in-Aid for the 21 century COE, "Center for Diversity and Universality in Physics". H.T. is supported by a Grant-in-Aid from the Ministry of Education, Science, Sports, and Culture of Japan.
${ }^{1}$ H. Kageyama, K. Yoshimura, R. Stern, N. V. Mushnikov, K. Onizuka, M. Kato, K. Kosuge, C. P. Slichter, T. Goto, and Y. Ueda, Phys. Rev. Lett. 82, 3168 (1999).

2 S. Miyahara and K. Ueda, Phys. Rev. B 61, 3417 (2000).

3 T. Momoi and K. Totsuka, Phys. Rev. B 62, 15067 (2000).

${ }^{4}$ K. Mitsuhira, Z. Hiroi, T. Tayama, S. Takagi, and T. Sakakibara, J. Phys.: Condens. Matter 14, L559 (2002).

5 M. Udagawa, M. Ogata, and Z. Hiroi, J. Phys. Soc. Jpn. 71, 2365 (2002).

${ }^{6}$ R. Moessner and S. L. Sondhi, Phys. Rev. B 68, 064411 (2003).

7 K. Hida, J. Phys. Soc. Jpn. 70, 3673 (2001).

8 M. E. Zhitomirsky, Phys. Rev. Lett. 88, 057204 (2002).

9 D. C. Cabra, M. D. Grynberg, P. C. W. Holdsworth, and P. Pujol, Phys. Rev. B 65, 094418 (2002).

10 J. Schulenburg, A. Honecker, J. Schnack, J. Richter, and
H.-J. Schmidt, Phys. Rev. Lett. 88, 167207 (2002).

11 J. Richter, J. Schulenburg, A. Honecker, J. Schnack, and H.-J. Schmidt, J. Phys.: Condens. Matter 16, S779 (2004).

12 J. Schnack, H.-J. Schmidt, J. Richter, and J. Schulenburg, Eur. Phys. J. B 24, 475 (2001).

13 H.-J. Schmidt, J. Phys. A 35, 6545 (2002).

14 A. P. Ramirez, B. S. Shastry, A. Hayashi, J. J. Krajewski, D. A. Huse, and R. J. Cava, Phys. Rev. Lett. 89, 067202 (2002).

15 G. Jackeli and M. E. Zhitomirsky, Phys. Rev. Lett. 93, 017201(2004).

16 M. E. Zhitomirsky, Phys. Rev. B 67, 104421 (2003).

17 S. S. Sosin, L. A. Prozorova, A. I. Smirnov, A. I. Golov, I. B. Berkutov, O. A. Petrenko, G. Balakrishnan, M. E. Zhitomirsky, e-print: cond-mat/0404529

18 A. P. Ramirez, G. P. Espinosa and A. S. Cooper, Phys. 
Rev. B 45, 2505 (1992).

19 K. Isawa, Y. Yaegashi, M. Komatsu, M. Nagano, S. Sudo, M. Karppinen, and H. Yamauchi, Phys. Rev. B 56, 3457 (1997).

20 M. E. Zhitomirsky and A. Honecker, J. Stat. Mech.: Theor.
Exp. P07012 (2004).

21 R. J. Baxter, Exactly Solved Models in Statistical Mechanics (Academic Press, London, 1982).

22 O. Derzhko and J. Richter, e-print: cond-mat/0404204 